\title{
DHA et développement du cerveau de l'enfant
}

\author{
Alexandre LAPILLONNE \\ Université René Descartes Paris $V$ et APHP, \\ Service de Néonatologie et Nutrition, \\ Hôpital Saint-Vincent de Paul, \\ 74 Avenue Denfert Rochereau, \\ 75014 Paris \\ <alexandre.lapillonne@svp.aphp.fr>
}

\begin{abstract}
N-3 Fatty acids are biologically important nutrients. One $n-3$ fatty acid, docosahexaenoic acid, is an important component of neural and retinal membranes and accumulates rapidly in the brain and retina during the later part of gestation and early postnatal life. It is reasonable to hypothesize that $n-3$ fatty acid intakes might have significant effects on infant visual function and neurodevelopmental status. Over the last two decades a lot of interest has been expressed about the long-chain polyunsaturated fatty acid (LCPUFA) requirements of both preterm and term infants. The aim of this review is to synthesize the recent data on the importance of the LCPUFA metabolism and early nutrition on infant growth and development.
\end{abstract}

Key words: newborns, DHA, growth, metabolism, neurological development, visual function

\section{Sources de DHA chez le nourrisson} gras polyinsaturés (AGPI) $\omega 3$ et $\omega 6$ a été 1 'un des domaines de recherche en nutrition pédiatrique les plus actifs au cours de ces deux dernières décennies car les AGPI à longue chaîne (AGPI-LC) tels que I'acide arachidonique (C20:4 106 ou ARA) et docosahexaénoïque (C22:603 ou $\mathrm{DHA})$ se sont révélés être des molécules indispensables à la croissance et au développement. Les membranes des cellules cérébrales, les jonctions synaptiques et les cellules à cônes et bâtonnets de la rétine sont particulièrement riches en $\mathrm{DHA}$, dérivé à longue chaîne de l'acide $\alpha$-linolénique.

Les études expérimentales ont montré qu'une carence en acides gras $\omega 3$ au cours des phases clés du développement, c'est-à-dire la vie fœtale et la vie post-natale immédiate, pouvait altérer significativement le développement des fonctions visuelles et cognitives chez l'animal [1]. L'ARA et l'acide eicosapentaénoïque (EPA ou C20:5(03), autres AGPI à longue chaîne, sont également importants car ce sont des précurseurs des prostaglandines, leucotriènes et thromboxanes, molécules regroupées sous le nom général d'eicosanoïdes. Chez les mammifères, la synthèse des AGPI à longue chaîne $\omega 3$ utilise le même système enzymatique que la synthèse des AGPI $\omega 6$ et $\omega 9$, ce qui rend l'organisme sensible à tout déséquilibre important entre les deux lignées.

La compréhension des adaptations physiologiques et du métabolisme chez le nouveau-né et le nourrisson est fondamentale pour pouvoir délivrer des conseils diététiques adaptés. Cet article de synthèse vise donc à présenter les données actuelles sur le rôle du métabolisme et de la nutrition des premiers mois de vie sur le développement et la croissance du nourrisson.
Le DHA s'accumule de façon préférentielle dans les membranes des cellules photoréceptrices de la rétine et des neurones où il y joue un rôle essentiel [1]. L'accumulation du DHA dans le tissu cérébral fœetal se fait essentiellement au cours du dernier trimestre de la grossesse, mais cette accumulation se poursuit en postnatal pendant les deux premières années de vie.

La source principale de DHA du fœtus provient du passage transplacentaire. Le statut en AGPI du nouveau-né à la naissance est bien corrélé avec le statut maternel mais est supérieur chez le nouveau-né montrant ainsi qu'il existe un ou plusieurs mécanismes conduisant à une accumulation préférentielle de certains acides gras pendant la vie fœtale [2]. Le placenta joue un rôle crucial en concentrant activement et en orientant spécifiquement les acides gras $\omega 3$ et $\omega 6$ vers le fœtus via des mécanismes multiples qui incluent une captation sélective par le syncytiotrophoblaste, un routage cellulaire spécifique et un relargage sélectif dans la circulation fœtale [3].

La couverture des besoins en AGPI-LC pendant les premiers mois de vie est assurée par le lait maternel car il contient naturellement des AGPI à longue chaîne. Si le lait maternel est le lait de référence du nourrisson pendant les 6 premiers mois de vie, sa composition est variable et dépend en partie de l'alimentation. En conséquence, les besoins du nourrisson ne peuvent pas être déduits par la simple extrapolation des quantités en AGPI du lait maternel. Toutefois, la compréhension de la physiologie du lait maternel peut nous donner des indications pour déterminer les besoins du nourrisson. Au cours de la lactation, la teneur en AGPI se modifie avec une augmentation de l'acide linoléique et de l'acide alphalinolénique entre le colostrum, le lait de transition et le lait mature alors que la teneur en AGPI-LC diminue [4]. Bien que sa composition change dans le temps et d'une femme à l'autre, le lait maternel contient toujours des AGPI-LC et en particulier de I'ARA et du DHA [4]. La teneur en AGPI-LC ne varie pas de façon totalement superposable selon que l'on considère les $\omega 6$ ou les $\omega 3$. En effet, des études relativement récentes utilisant les isotopes stables montrent que $90 \%$ de l'acide arachidonique du lait maternel provient de la mobilisation des pools maternels, essentiellement le tissu adipeux et le plasma [5]. Cette contribution importante du tissu adipeux et des autres réserves maternelles peut en fait être biologiquement bénéfique pour l'enfant car elle assure un apport relativement stable en cet AGPI même si le régime de la mère change sur une courte période. La concentration en acide arachidonique du lait maternel est donc relativement constante d'une femme à l'autre.

À l'opposé, les concentrations en DHA du lait maternel montrent des variations considérables et dépendent en grande partie de l'alimentation de la mère [4]. Des études randomisées de supplémentation maternelle ont clairement montré un effet direct de la supplémentation sur les concentrations en DHA du lait maternel [6] et les études avec les isotopes stables montrent la proportion de DHA issu de l'alimentation et transféré dans le lait maternel est constante quelle que soit la quantité de DHA présente dans I'alimentation [7]. L'ensemble de ces données montre que l'apport en DHA de I'enfant allaité est directement dépendant de la consommation maternelle d'aliments riches en AGPI-LC $\omega 3$ comme les huiles de poissons. Les taux d'AGPI à longue chaîne dans le lait maternel sont en moyenne, en Europe, de 0,3\% pour le DHA et de 0,5\% pour l'ARA [8].

Au contraire du lait maternel, les formules de lait infantile ne contiennent pas de DHA et d'ARA et le nouveau-né recevant ce type de lait artificiel non supplémenté est donc totalement dépendant de ses capacités de synthèse endogène et de mobilisation de ses réserves. Le 
nouveau-né est capable de synthétiser des AGPI à longue chaîne à partir des précurseurs [9] mais nous savons que cette synthèse est probablement insuffisante pour assurer la couverture des besoins en DHA et ARA au cours de la phase de croissance rapide de la première année. En effet, après plusieurs mois d'alimentation avec un lait infantile non supplémenté, les nourrissons présentent non seulement une diminution significative du contenu en DHA des lipides plasmatiques ou des phospholipides des membranes des globules rouges, deux paramètres étant habituellement utilisés pour apprécier le statut en AGPI d'un enfant, mais également une plus faible concentration en DHA cérébral par rapport aux enfants allaités [1]. L'accumulation en DHA entre la naissance et 6 mois est en fait négative chez l'enfant nourri au lait artificiel, I'accumulation de DHA cérébral ne se faisant qu'au prix d'une mobilisation importante des réserves hépatiques et du tissu adipeux [10]. L'apport d'AGPI à longue chaîne préformés dans l'alimentation du nourrisson au cours de la première année de vie est donc nécessaire pour assurer un statut en AGPI comparable à celui observé chez le nourrisson allaité.

\section{Études fonctionnelles chez le nouveau-né à terme}

La question essentielle est de savoir si ces modifications biochimiques ont un impact significatif sur le développement neurosensoriel des enfants. La comparaison du développement neurosensoriel de l'enfant allaité avec celui de l'enfant recevant un lait artificiel ne peut en aucun cas permettre de déterminer si le DHA joue un rôle dans le développement neurosensoriel tant les différences d'ordre nutritionnel et d'ordre non nutritionnel sont importantes entre ces deux groupes d'enfants. Par contre, deux études récentes ont comparé parmi les enfants allaités, ceux qui recevaient un lait maternel naturellement riche en DHA avec ceux qui recevaient un lait maternel naturellement plus pauvre en DHA. Bien qu'il existe de nombreuses limitations à ces études, elles montrent une association significative entre le contenu en DHA du lait maternel et l'acuité visuelle à l'âge de 2 mois et 12 mois dans une étude [11] et à l'âge de 4 mois dans l'autre [12].

Les données les plus solides concernant l'importance de l'apport en DHA préformé sur le développement du nourrisson ne peuvent provenir que des études randomisées en double aveugle dans lesquelles un groupe d'enfants reçoit un lait artificiel standard et un autre un lait artificiel supplémenté en DHA [13]. Toutes les études de supplémentation ont montré leurs efficacités pour normaliser le sta- tut en DHA du nouveau-né à terme par rapport au nouveau-né allaité. Pendant longtemps, les résultats des études de supplémentation ont semblé conflictuels au regard du développement neurosensoriel avec autant d'études montrant un effet positif significatif de la supplémentation en DHA que d'études ne montrant pas d'effet. Cette variabilité dans les résultats des études de supplémentation peut s'expliquer par l'hétérogénéité des sources, de la durée et des quantités d'AGPI utilisées, de la teneur en précurseurs, des tests utilisés, et de l'origine ou le niveau socio-économique de la population étudiée.

Une méta-analyse récente montre une meilleure maturation visuelle appréhendée par les méthodes comportementales à deux mois de vie mais pas ultérieurement, chez les enfants nés à terme ayant reçu un lait artificiel supplémenté en DHA par rapport aux enfants ayant reçu un lait non supplémenté [14]. Récemment, les données de ces études de supplémentation ont été réévaluées afin de tenir compte non seulement du DHA préformé apporté par la supplémentation mais également du DHA synthétisé par l'organisme à partir de l'acide alphalinolénique [15]. Cette nouvelle approche montre qu'il existe une corrélation significative entre la dose calculée de DHA (préformé + synthétisé) et l'acuité visuelle à l'âge de 4 mois.

Les études de supplémentation visant à étudier le développement neurologique des nourrissons ont donné des résultats aussi hétérogènes que les précédentes mais il semble que la variabilité des résultats puisse également, au moins en partie, s'expliquer par la quantité de DHA utilisée pour la supplémentation, une supplémentation avec $0,36 \%$ des acides gras sous forme de DHA et $0,72 \%$ sous forme d'ARA améliorant le développement moteur évalué par le score de Bayley à 18 mois [16] alors qu'une supplémentation avec $0,14 \%$ des acides gras sous forme de DHA et $0,46 \%$ sous forme d'ARA s'est montrée dépourvue d'efficacité sur ce score à 1 an [13].

Sur la base de ces données, un groupe d'experts, spécialistes des AGPI, recommande que les formules de lait artificiel pour enfant à terme contiennent des taux de DHA et d'ARA proches de ceux observés en moyenne dans le lait maternel (au moins 0,2\% des acides gras sous forme de DHA et $0,35 \%$ sous forme d'ARA) [17].

\section{Études fonctionnelles chez le nouveau-né prématuré}

Les effets bénéfiques sur le développement neurosensoriel d'une supplémentation en DHA dans les formules de lait artificiel sont admis depuis plusieurs années chez l'enfant préma- turé [13] et les laits artificiels pour enfants de faible poids de naissance sont tous, en Europe, enrichis en DHA. Toutefois, il y a eu pendant longtemps une certaine réticence à utiliser un lait artificiel enrichi en AGPI 103 en raison de I'observation, dans l'une des premières études, d'une croissance staturo-pondérale inférieure chez les enfants prématurés ayant reçus un lait artificiel enrichi exclusivement en AGPI $\omega 3$ par rapport au groupe ayant reçu un lait standard. Un déséquilibre entre les deux lignées d'AGPI $\omega 3$ et $\omega 6$, résultant du rajout exclusif d'AGPI $\omega 3$ (DHA et EPA) sans ajout concomitant d'AGPI 16 (ARA) est l'un des mécanismes pouvant expliquer cette observation [18]. À I'inverse, toutes les études utilisant une supplémentation combinée en DHA et ARA se sont avérées parfaitement adaptées pour assurer une croissance optimale $[19,20]$.

Bien que la plupart des essais cliniques de supplémentation chez le prématuré aient assuré une supplémentation pendant la première année de vie, les anciens prématurés ne reçoivent en pratique clinique qu'une supplémentation de quelques semaines ou de quelques mois car les laits artificiels utilisés en relais du lait pour enfants de faible poids de naissance ne contiennent pas d'AGPI à longue chaîne. Un lait premier et deuxième âge supplémenté en AGPI à longue chaîne serait essentiel pour cette catégorie d'enfants à haut risque neurologique.

\section{Supplémentation en DHA après le sevrage du lait maternel}

La question du sevrage est également une question très importante, en particulier en France où la durée de l'allaitement est relativement courte. Nous avons montré que le statut en DHA des nourrissons à l'âge de 4 mois est corrélé positivement avec la durée d'allaitement maternel pendant les premiers mois de vie [21]. Deux études de supplémentation réalisées après le sevrage du lait maternel documentent l'intérêt d'un lait enrichi en AGPI à longue chaîne au moment du sevrage [22, 23]. Ces 2 études montrent qu'après un sevrage réalisé à 6 semaines ou entre 4 et 6 mois la poursuite d'une formule supplémentée avec des concentrations en DHA et ARA proches des concentrations observées dans le lait maternel $(0,36 \%$ de DHA et $0,72 \%$ d'ARA ) maintient plus longtemps un statut en DHA proche de celui des enfants allaités mais, également, permet d'améliorer la fonction visuelle à 17, 26 et 52 semaines dans une étude et à 1 an dans l'autre étude [22, 23]. Ces études plaident donc en faveur du choix d'un lait artificiel enrichi en DHA et ARA au moment du sevrage du lait maternel. Elles suggèrent également qu'un 
apport en DHA et ARA préformé puisse être important pendant l'ensemble de la première année de vie.

\section{Conclusion}

Les acides gras $\omega 3$ et $\omega 6$ sont des nutriments biologiquement importants en particulier pour le développement du nourrisson. Le statut maternel pendant la grossesse et la lactation sont essentiels pour assurer au fœtus et au nouveau-né un apport en acides gras $\omega 3$ et $\omega 6$ optimal. La femme enceinte et la femme allaitante doivent donc respecter les apports nutritionnels conseillés (ANC) aussi bien en précurseurs qu'en AGPI-LC. L'allaitement maternel est l'alimentation à préférer pour un enfant sain et qui doit être fortement encouragé, I'Organisation mondiale de la santé (OMS) recommandant un allaitement exclusif pendant 6 mois. En cas d'impossibilité d'allaitement maternel, mais également en relais de l'allaitement maternel, prescrire un lait artificiel enrichi en DHA et ARA chez l'enfant à terme et chez I'enfant prématuré paraît souhaitable au regard des données actuelles de la littérature.

\section{RÉFÉRENCES}

1. LAURITZEN L, HANSEN HS, JORGENSEN MH, MICHAELSEN KF. The essentiality of long chain $\mathrm{n}-3$ fatty acids in relation to development and function of the brain and retina. Prog Lipid Res $2001 ; 40: 1-94$

2. AL MDM, VAN HOUWELINGEN AC, KESTER ADM, HASAART HTM, DE JONG AEP, HORNS TRA G. Maternal essential fatty acid patterns during normal pregnancy and their relationships with the neonatal essential fatty acid status. Br / Nutr 1995 ; 74 : 55-68.

3. HAGGARTY P. Effect of placental function on fatty acid requirements during pregnancy. Eur Clin Nutr $2004 ; 58: 1559-70$.

4. JENSEN RG. Lipids in human milk. Lipids 1999 ; 34 : 1243-71.

5. DEL PRADO M, VILLALPANDO S, ELIZONDO A, RODRIGUEZ M, DEMMELMAIR H, KOLETZKO B. Contribution of dietary and newly formed arachidonic acid to human milk lipids in women eating a low-fat diet. Am / Clin Nutr 2001 ; 74 : 242-7.
6. JENSEN CL, MAUDEM, ANDERSON RE, HEIRD WC. Effect of docosahexaenoic acid supplementation of lactating women on the fatty acid composition of breast milk lipids and maternal and infant plasma phospholipids. Am J Clin Nutr 2000 ; 71(1 Suppl) : 292S-299S.

7. FIDLER N, SAUERWALD T, POHL A, DEMMELMAIR H, KOLETZKO B. Docosahexaenoic acid transfer into human milk after dietary supplementation : a randomized clinical trial. / Lipid Res $2000 ; 41: 1376-83$.

8. KOLETZKO B, THIEL I, ABIODUN PO. The fatty acid composition of human milk in Europe and Africa. / Pediatr 1992; 120 : S62-S70.

9. SAUERWALD TU, HACHEY DL, JENSEN CL, CHEN H, ANDERSON RE, HEIRD WC. Intermediates in endogenous synthesis of C22:6 omega 3 and C20:4 omega 6 by term and preterm infants. Pediatr Res 1997 ; 41 : 183-7.

10. CUNNANE SC, FRANCESCUTTI V, BRENNA JT, CRAWFORD MA. Breast-fed infants achieve a higher rate of brain and whole body docosahexaenoate accumulation than formula-fed infants not consuming dietary docosahexaenoate. Lipids $2000 ; 35$ : 105-11.

11. INNIS SM, GILLEY J, WERKER J. Are human milk long-chain polyunsaturated fatty acids related to visual and neural development in breast-fed term infants? J Pediatr $2001 ; 139$ : 532-8.

12. JORGENSEN MH, HERNELL $O$, HUGHESE, MICHAELSEN KF. Is there a relation between docosahexaenoic acid concentration in mothers' milk and visual development in term infants? J Pediatr Gastroenterol Nutr 2001 ; 32 : 293-6.

13. HEIRD WC, LAPILLONNE A. The role of essential fatty acids in development. Annu Rev Nutr $2005 ; 25: 549-71$

14. SANGIOVANNI JP, BERKEY CS, DWYER JT, COLDITZ GA. Dietary essential fatty acids, long-chain polyunsaturated fatty acids, and visual resolution acuity in healthy fullterm infants: a systematic review. Early Hum Dev $2000 ; 57: 165-88$.

15. UAUY R, HOFFMAN DR, MENA P, LLANOS A, BIRCH EE. Term infant studies of DHA and ARA supplementation on neurodevelopment: results of randomized controlled trials. J Pediatr 2003 ; 143 : S17-S25.
16. BIRCH EE, GAFIELD SHDR, UAUY R, BIRCH DG. A randomized controlled trial of early dietary supply of long-chain polyunsaturated fatty acid and mental develoment in term infants. Dev Med Child Neurol $2000 ; 42$ : 174-81.

17. KOLETZKO B, AGOSTONIC, CARLSON SE, $E T A L$. Long chain polyunsaturated fatty acids (LC-PUFA) and perinatal development. Acta Paediatr $2001 ; 90$ : 460-4.

18. LAPILLONNE A, CLARKE SD, HEIRD WC. Plausible mechanisms for effects of long-chain polyunsaturated fatty acids on growth. I Pediatr $2003 ; 143$ : S9-S16.

19. CLANDININ MT, VAN AERDE JE, MERKEL KL, ET AL. Growth and development of preterm infants fed infant formulas containing docosahexaenoic acid and arachidonic acid. / Pediatr $2005 ; 146: 461-8$.

20. GROH-WARGO S, JACOBS J, AUESTAD N, O'CONNOR DL, MOORE IJ, LERNER E. Body composition in preterm infants who are fed long-chain polyunsaturated fatty acids : a prospective, randomized, controlled trial. Pediatr Res $2005 ; 57: 712-8$.

21. LAPILLONNE A, BROSSARD N, CLARIS O, REYGROBELLET B, SALLE BL. Erythrocyte fatty acid composition in term infants fed human milk or a formula enriched with a low eicosapentanoic acid fish oil for 4 months. Eur I Pediatr $2000 ; 159: 49-53$.

22. BIRCH EE, HOFFMAN DR, CASTANEDA YS, FAWCETT SL, BIRCH DG, UAUY RD. A randomized controlled trial of long-chain polyunsaturated fatty acid supplementation of formula in term infants after weaning at $6 \mathrm{wk}$ of age. Am I Clin Nutr 2002 ; 75 : 570-80.

23. HOFFMAN DR, BIRCH EE, CASTANEDA YS, ET AL. Visual function in breast-fed term infants weaned to formula with or without long-chain polyunsaturates at 4 to 6 months : a randomized clinical trial. / Pediatr $2003 ; 142$ : 669-77. 